\title{
CLASSIFICAÇÃO DA SUSTENTABILIDADE DAS UNIDADES DE PRODUÇÃO AGRÍCOLA NO PERÍMETRO IRRIGADO ARARAS NORTE, CEARÁ
}

\section{SUSTAINABILITY CLASSIFICATION OF AGRICULTURAL PRODUCTION UNITS IN THE ARARAS NORTE IRRIGATED DISTRICT, CEARÁ, BRAZIL ${ }^{1}$}

\author{
Eunice Maia de ANDRADE ${ }^{2}$ \\ José Alves CARNEIRO NETO ${ }^{3}$ \\ Morsyleide de Freitas ROSA ${ }^{4}$ \\ Helba Araújo Queiroz PALÁCIO ${ }^{5}$ \\ Joseilson Oliveira RODRIGUES ${ }^{6}$
}

\begin{abstract}
RESUMO
Este estudo teve como objetivo identificar os fatores determinantes da sustentabilidade das unidades de produção agrícola do Perímetro Irrigado Araras Norte, utilizando um índice de sustentabilidade pelo emprego de estatística multivariada. Os dados empregados nesta pesquisa foram originários de questionários aplicados a 47 irrigantes do Perímetro Irrigado Araras Norte, localizado na parte alta da bacia do rio Acaraú, Ceará, Brasil. De acordo com os resultados obtidos, o nível sócio-educacional das famílias irrigantes foi identificado como o fator de maior peso na definição da sustentabilidade das unidades de produção agrícola do Perímetro Irrigado Araras Norte. O segundo fator foi determinado por variáveis relacionadas com o processo de comercialização (dificuldades de venda e transporte da produção); enquanto que o terceiro foi definido por variáveis que expressam a situação da agricultura familiar (participação dos filhos e o interesse desses em dar continuidade às atividades agrícolas). O último fator foi apontado por variáveis relacionadas com as técnicas de conservação dos recursos naturais. $O$ índice de sustentabilidade desenvolvido a partir dos fatores determinantes selecionados registrou que $27,7 \%$ das unidades de produção agrícola foram classificadas com sustentáveis, $25,5 \%$ apresentaram a sustentabilidade ameaçada e $14,9 \%$ sustentabilidade comprometida. Das 47 unidades, 11 foram classificadas como insustentáveis ou seriamente insustentáveis, o que corresponde a $23,4 \%$.
\end{abstract}

Palavras-chave: agricultura irrigada; análise fatorial; índice de sustentabilidade.

\begin{abstract}
The aim of this study was to identify the determinant sustainability factors in agricultural production units in the Araras North Irrigated District, as well as to develop a sustainability index applying multivariate statistical technique. The approach was based on a cross-over form applied to 47 irrigators that live in the Araras North Irrigated District located in the uplands of the Acaraú watershed, Ceará, Brazil. According to the results the family education level was the major factor in determining the sustainability of the agricultural production units in the Araras North Irrigated District. The second factor was mainly related to the trade processes (selling difficulties and production transportation); while the third one was defined by the variables that express the irrigator's family condition (the youth of the community involvement and their interest in continuing the agricultural activities). The last factor was pointed out by variables related to natural resources conservation technique. The developed Sustainability Index from selected determinant factors registered that $27.7 \%$ of the agricultural production units were classified as sustainables, $25.5 \%$ presented a threatened sustainability and $14.9 \%$ had a compromised sustainability. Among the 47 units, 11 were classified as unsustainable, which represents $23.4 \%$ of them.

Key-words: irrigated agriculture; factorial analysis; sustainability index.
\end{abstract}

${ }^{1}$ Parte da dissertação de mestrado do segundo autor apresentado ao mestrado de Irrigação e Drenagem do Departamento de Engenharia Agrícola da Universidade Federal do Ceará (UFC), CE.

${ }^{2}$ Eng. Agrônoma, Phd., Professora do Departamento de Engenharia Agrícola, Centro de Ciências Agrárias (CCA)/Universidade Federal do Ceará - Av. Mister Hull S/N, Bloco 804, 60455-970, Fortaleza, CE - Brasil. E-mail: eandrade@ufc.com.br. Autor para correspondência.

${ }^{3}$ Eng. Civil, Mestre em Agronomia. Fortaleza, CE - Brasil. E-mail: alvesneto@superig.com.

${ }^{4}$ Eng. Química, Doutora em Tecnologia de Processos Químicos e Bioquímicos, Pesquisadora da Embrapa Agroindústria Tropical, Fortaleza - CE - Brasil. E-mail: morsy@cnpat.embrapa.br.

${ }^{5}$ Licenciada em Ciências Agrárias, Doutoranda em Agronomia, Professora da Escola Agrotécnica Federal de Iguatu, CE - Brasil. E-mail: gabinete@eafigt.gov.br.

${ }^{6}$ Eng.Agrônomo, Mestrando em Irrigação e Drenagem, bolsista do CNPq. Fortaleza, CE - Brasil. E-mail: wilson_agronomia@hotmail.com. 
ANDRADE, E.M. et al. Classificação da sustentabilidade das unidades de produção...

\section{INTRODUÇÃO}

Os cientistas da área agrícola e os produtores rurais têm como desafio o desenvolvimento de um novo paradigma para a agricultura, o qual represente o conceito de sustentabilidade (Sands \& Podmore, 2000). Todavia, embora a sustentabilidade seja vista como um tema de primordial importância para a vida do planeta, a agricultura sustentável continua como uma concepção não muito clara (Mangabeira et al., 2002). A atual tendência que envolve o conceito de desenvolvimento sustentável implica no aproveitamento racional dos recursos naturais com base na capacidade de suporte do ambiente. Porém, agregados aos problemas ambientais, existem os desafios sócio-econômicos, geralmente caracterizados pela operacionalização dos produtores agrícolas (Melo, 1999; Luiz \& Silveira, 2000)

Um dos desafios enfrentados pela discussão sobre desenvolvimento sustentável é a elaboração de metodologias aplicadas que permitam avaliar a sustentabilidade de diferentes projetos, tecnologias ou agroecossistemas em situações concretas (Masera et al., 1999). Este desafio é definido, em especial, pela necessidade de questionamento das formas convencionais de avaliar esses projetos, tecnologias e sistemas de manejo de recursos naturais. Com relação à sustentabilidade dos pequenos produtores da agricultura irrigada, por exemplo, existem relatos de que esta depende do nível educacional dos referidos produtores, das condições climáticas locais, do uso da terra, da geologia, da qualidade da água de irrigação, da drenagem natural do solo e da comercialização, entre outros (Marzall \& Almeida, 1998; Melo, 1999; Souza et al., 2001; Castelo Branco, 2003).

Toda essa complexidade torna difícil estabelecer uma única variável como um indicador padrão em qualquer sistema de produção agrícola. Nesta busca, técnicas de estatística multivariada, como a Análise Fatorial/Análise da Componente Principal, vem sendo empregadas no desenvolvimento de índices de sustentabilidade que possam refletir resumidamente as alterações sofridas pelo meio ambiente em decorrência do sistema adotado pelo produtor agrícola: agricultura irrigada ou de sequeiro (Gallopín, 1997; Andrews et al., 2002; Palácio, 2004). Um índice ou indicador é uma ferramenta que permite a obtenção de informações sobre uma dada realidade, sendo como principal característica o poder de sintetizar um conjunto complexo de informações, retendo apenas o significado essencial dos aspectos analisados (Silveira \& Andrade, 2002; Fossatti \& Freitas, 2004). Este estudo teve como objetivo identificar os fatores determinantes da sustentabilidade do Perímetro Irrigado Araras Norte, utilizando um índice de sustentabilidade pelo emprego da estatística multivariada.

\section{MATERIALE MÉTODOS}

A área em estudo, o Perímetro Irrigado Araras Norte, abrange parte da área dos municípios de Varjota e Reriutaba, localizados na região norte do Estado do Ceará (Figura 1). O referido perímetro fica situado a jusante do açude Paulo Sarasate, o qual possui uma área irrigada de $1.345,80$ ha. A implantação do perímetro irrigado foi iniciada em 1987, e os serviços de administração, operação e manutenção da infra-estrutura de uso comum tiveram início no ano de 1998.

O universo dos irrigantes que exploram o perímetro irrigado Araras Norte, subdivididos por categoria, dimensão do lote unitário, total de unidades similares e área total correspondente a cada categoria estão presentes na Tabela 1. Durante a realização dessa pesquisa, os lotes empresariais (605,8 ha) e mais 150 ha distribuídas entre as outras categorias de irrigantes se encontravam inoperantes, as quais representam $56 \%$ da área do perímetro irrigado Araras Norte. Conforme análise preliminar dos dados de campo, os colonos entrevistados possuem, em média, cerca de 10 ha irrigados. É possível constatar a presença de unidades de produção agrícola com maior porte devido à comercialização das unidades de desistência entre os próprios titulares.

O suprimento hídrico do perímetro ocorre através de uma vazão contínua de aproximadamente $2,1 \mathrm{~m}^{3} \mathrm{~s}^{-1} \mathrm{ha}^{-1}$ por produtor, liberada pelo Açude Público Federal Paulo Sarasate. A rede de adução, responsável pela condução de água desde o açude até cada um dos setores, é composta de tubulações de recalque e adução, com uma rede de canais. $O$

TABELA 1 - Classificação dos irrigantes e dimensões dos lotes que compõem o Perímetro Irrigado Araras Norte, Ceará, 2004.

\begin{tabular}{cccc}
\hline $\begin{array}{c}\text { Categorias de } \\
\text { irrigantes }\end{array}$ & $\begin{array}{c}\text { Área média } \\
\text { (ha) }\end{array}$ & $\begin{array}{c}\text { Quantidade } \\
\text { (unid.) }\end{array}$ & $\begin{array}{c}\text { Área total } \\
\text { (ha) }\end{array}$ \\
\hline Pequeno Produtor & 7,00 & 89 & 623,00 \\
Técnico Agrícola & 11,00 & 3 & 33,00 \\
Eng $^{\circ}$ Agrônomo & 9,33 & 9 & 84,00 \\
Empresa & 302,90 & 2 & 605,80 \\
Total & & 103 & $1.345,80$ \\
\hline
\end{tabular}

Fonte: Departamento Nacional de Obras Contra as Secas (DNOCS), 2005. 


\section{$41^{\circ} 0^{\prime} \mathrm{O}$ "W $\quad 40^{\circ} 30^{\prime} \mathrm{O}^{\prime \prime} \mathrm{W} \quad 40^{\circ} 00^{\prime} 0^{\prime \prime} \mathrm{W} \quad 39^{\circ} 30^{\prime} 0^{\prime \prime} \mathrm{W}$}

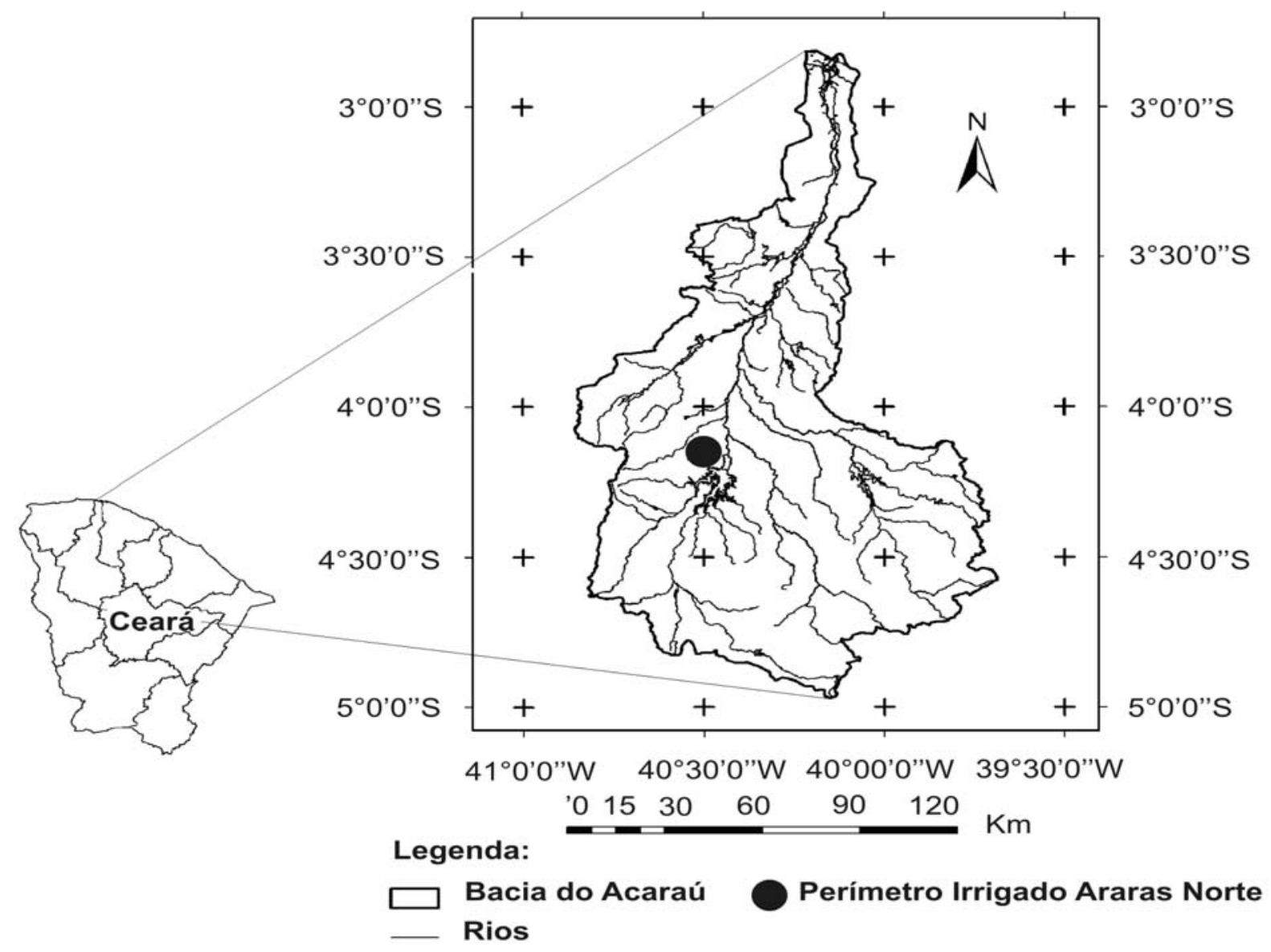

FIGURA 1 - Localização do Perímetro Irrigado Araras Norte no Estado do Ceará.

projeto também possui um reservatório de compensação, cuja função é armazenar o volume d'água bombeado pela estação principal durante as horas de paralisação da operação do sistema. O sistema de drenagem é constituído por drenos e valas naturais, com vazões variáveis.

As unidades de produção agrícola do perímetro irrigado Araras Norte que se encontram em plena atividades são exploradas em quase sua totalidade com as culturas do mamão, o coco, a banana e a uva, sendo essa última voltada para a exportação. Os sistemas de irrigação empregados no perímetro são a aspersão convencional $(89,44 \%$ da área) e micro-aspersão $(10,56 \%$ da área).

O clima da região, segundo a classificação de Koeppen, é do tipo BSw'h' - semiárido quente, caracterizado por duas estações distintas: uma estação chuvosa, com precipitações irregulares e outra seca. O perímetro irrigado Araras Norte encontra-se principalmente sobre solos classificados como Luvissolos e, uma parcela mínima (2\%) sobre os Latossolos (DNOCS, 1977). A coleta de dados, através da aplicação de questionário aos irrigantes (47 no total) os quais representam as unidades de produção agrícola, foi realizada em julho de 2004, quando se iniciam as atividades de irrigação nos perímetros. Tratava-se de um questionário de elaboração própria com perguntas objetivas e fechadas obtendo respostas diretas, de forma a padronizar os dados. Previamente foram contactadas as associações e as cooperativas de irrigantes, bem como as sedes regionais do Departamento Nacional de Obras Contra a Seca (DNOCS) nas áreas visitadas, de forma que os indicadores selecionados para avaliação de eficiência fossem obtidos a partir das críticas realizadas pelos atores sociais, e um extensivo conjunto obtido na revisão de literatura. O subconjunto de indicadores adotados na avaliação de eficiência foi selecionado objetivando assegurar representação de resultados econômicos, e de bem-estar social, em resposta à utilização dos recursos natural/área agricultável, trabalho (mão-deobra), capital e tecnologia (tratores e tração animal). Foram realizadas 44 perguntas (variáveis) nas quais se abordou os aspectos sócio-econômicos, agronômicos (uso e ocupação do solo, aplicação de técnicas de manejo e conservação, contaminação de recursos hídricos e lixo) e da rede de saúde e lazer. Aspectos como a visão do produtor sobre a sua propriedade, a agricultura familiar, a sucessão da propriedade e necessidade de treinamento também foram abordadas junto aos produtores. 
A seleção dos fatores determinantes da sustentabilidade do perímetro irrigado Araras Norte fundamentou-se na aplicação do modelo de estatística multivariada Análise Fatorial/Análise das Componentes Principais (AF/ACP). Nesta análise, cada uma das $n$ variáveis forma uma combinação linear de $m$ fatores comuns e de um fator específico, sendo que o número de fatores comuns deve ser inferior ao número de variáveis. Essa metodologia é composta pelas seguintes etapas: preparação da matriz de correlação; extração dos fatores comuns com possível redução dos parâmetros que definem a sustentabilidade ambiental; rotação dos eixos relativos aos fatores comuns, visando uma solução de mais fácil interpretação. Nesse estudo a matrix de correlação era composta por 44 colunas (variáveis) e 47 linhas (unidades de produção agrícola). Maiores informações podem ser obtidas em Dillon \& Goldstein (1984); Palácio (2004); Hair Junior et al. (2005); Girão et al. (2007), Andrade et al. (2009).

Tendo-se por base a seleção dos fatores determinantes elaborou-se $o$ índice de sustentabilidade (IS) o qual é definido pelo somatório do produto entre o escore de cada variável $\left(I_{i}\right)$ atribuído a cada unidade produtiva e o termo de ponderação dos indicadores no índice $\left(\mathrm{p}_{\mathrm{i}}\right)$. A expressão algébrica do índice é dada por:

$$
\text { IS }=p_{1} l_{1}+p_{2} I_{2} \ldots+p_{i} I_{i}
$$

Os escores aplicados a cada variável (li) foram fundamentados em Barreto et al. (2005). Já o valor do peso (pi) atribuído a variável foi ponderado em função do autovalor da componente (raiz característica) associado à explicabilidade de cada variável, em relação às componentes principais extraídas (Equação 2). O autovalor é utilizado como termo de ponderação por expressar a capacidade dos fatores em captar em níveis diferentes as variâncias das variáveis (Palácio, 2004).

$$
\mathrm{p}_{\mathrm{i}}=\frac{\left(\mathrm{F}_{1} \cdot \mathrm{P}_{\mathrm{i}}\right)+\left(\mathrm{F}_{2} \cdot \mathrm{P}_{\mathrm{i}}\right)}{\left(\mathrm{F}_{1} \cdot \sum_{1}^{\mathrm{n}} \mathrm{P}_{\mathrm{i}}\right)+\left(\mathrm{F}_{2} \cdot \sum_{1}^{\mathrm{n}} \mathrm{P}_{\mathrm{i}}\right)}
$$

onde: $\mathrm{p}_{\mathrm{i}}$ : peso a ser associado aos parâmetros de sustentabilidade; F: autovalor das componentes principais; $P_{i}$ : explicabilidade de cada variável em relação a componente principal. Uma vez que o índice, potencialmente, pode variar entre 0 e 1, optou-se por fundamentar a divisão de classes nos de Melo (1999). As unidades de produção agrícola foram classificadas da seguinte forma: Sustentável: IS < 0,20; Sustentabilidade Ameaçada; $0,20 \leq$ IS $<0,40$; Sustentabilidade Comprometida: $0,40 \leq$ IS <0,60; Insustentável: $0,60 \leq$ IS $<0,80$ e Seriamente Insustentável: IS $\geq 0,80$.

\section{RESULTADOS E DISCUSSÃO}

O emprego da análise fatorial pelo método dos componentes principais reduziu o universo das
44 variáveis iniciais a 13 variáveis agrupadas em cinco componentes com raízes características superiores à unidade, e que, em conjunto, explicaram $67,27 \%$ da variância total das variáveis selecionadas (Tabela 2).

Pode-se observar que a comunalidade de cada variável foi superior a 0,5 ; ou seja, as componentes reproduziram mais da metade da variância da referida variável (Hair Junior et al., 2005). Dos $67,2 \%$ da variância total explicada pelo modelo, $33 \%$ se concentra nas duas primeiras componentes, demonstrando que a sustentabilidade agroambiental apresenta dependência diferenciada das componentes definidas pelo modelo aplicado (Sands \& Podmore, 2000; Andrews et al., 2002).

Conceitua-se como fator o aspecto que é identificado pelo conjunto de variáveis de maior carga presentes em cada componente. Pela Tabela 2 pode-se observar que as variáveis de maior carga $(>0,500)$ para o componente 1 são indicadores do nível intelectual e social das famílias dos irrigantes, sendo portanto definido como o fator "Nível social e educacional da família". Fossatti \& Freitas (2004) identificaram variáveis semelhantes como determinantes das características sociais e econômicas da agricultura familiar no município de Santa Cruz (RS).

O componente 2 (Tabela 2) apresentou maior relação (carga $>0,580$ ) com as variáveis: "tempo de exploração da propriedade", "problemas por ocasião da comercialização" e "possui veículo para transporte da produção" expressando um fator de "processo da comercialização". A pesquisa identificou em campo, que a disponibilidade de pelo menos um carro com carroceria que facilitasse o escoamento da produção seria um aspecto relevante para o produtor. O escoamento da produção individual ou em conjunto está associada a condição financeira dos irrigantes, estando os produtores de menor poder econômico totalmente dependentes dos atravessadores. Os irrigantes foram unânimes em afirmar que as maiores dificuldades com a comercialização da produção foram "preços baixos" e os "atravessadores".

Os componentes 3, 4 e 5 apresentam-se como indicadores de um modelo de agricultura familiar em crise e a conservação dos recursos solo e água, originando assim um fator denominado de "Agricultura familiar" e um outro de "Adoção de técnicas de conservação". Através da Tabela 2 observa-se que as variáveis "continuidade das atividades agrícolas", "os filhos participam das atividades agrícolas" e "prestação de serviços temporários" apresentam cargas elevadas $(-0,881$; $-0,769$ e - $-0,616)$ expressando a desinteresse pela atividade agrícola exercida. Acredita-se que esta falta de motivação em dar continuidade à agricultura familiar seja decorrente da dificuldade de comercialização do produto agrícola e a oferta de empregos na indústria no município de Sobral/CE, a qual fica a uma distância inferior a $100 \mathrm{~km}$ deste perímetro irrigado. Este aspecto é preocupante, pois mostra uma perspectiva financeira desassociada 
ANDRADE, E.M. et al. Classificação da sustentabilidade das unidades de produção...

TABELA 2 - Cargas apresentadas pelas variáveis em cada componente que define os fatores determinantes da sustentabilidade das unidades de produção agrícola. Perímetro Irrigado Araras Norte, Ceará, 2004.

\begin{tabular}{|c|c|c|c|c|c|c|c|}
\hline \multirow{2}{*}{$\mathrm{N}^{\circ}$} & \multirow{2}{*}{ Variáveis } & \multicolumn{6}{|c|}{ Componentes } \\
\hline & & C1 & $\mathrm{C} 2$ & C3 & $\mathrm{C} 4$ & C5 & $\mathrm{C}^{*}$ \\
\hline 1 & Tamanho da família & 0,786 & 0,123 & 0,430 & 0,223 & $-0,124$ & 0,883 \\
\hline 2 & Número de filhos & 0,786 & 0,246 & 0,390 & 0,111 & $-0,089$ & 0,851 \\
\hline 3 & Treinamento que gostaria de receber & 0,768 & $-0,056$ & $-0,234$ & $-0,152$ & 0,113 & 0,683 \\
\hline 4 & Grau de Instrução & $-0,511$ & 0,108 & $-0,037$ & $-0,392$ & 0,510 & 0,688 \\
\hline 5 & Tempo de exploração da propriedade & $-0,121$ & 0,776 & $-0,101$ & $-0,041$ & 0,023 & 0,630 \\
\hline 6 & Problemas por ocasião da comercialização & $-0,075$ & $-0,750$ & 0,152 & $-0,123$ & 0,012 & 0,607 \\
\hline 7 & Possui veículo para transporte da produção & $-0,281$ & $-0,587$ & $-0,164$ & 0,093 & $-0,084$ & 0,466 \\
\hline 8 & Continuidade das atividades agrícolas & 0,004 & $-0,024$ & $-0,881$ & $-0,050$ & 0,078 & 0,786 \\
\hline 9 & Os filhos participam das atividades agrícolas & 0,175 & $-0,088$ & $-0,769$ & 0,098 & $-0,150$ & 0,662 \\
\hline 10 & Práticas agrícolas & 0,037 & $-0,091$ & $-0,039$ & 0,834 & 0,095 & 0,716 \\
\hline 11 & Sinais de poluição das fontes hídricas & 0,037 & 0,474 & 0,205 & 0,567 & $-0,008$ & 0,590 \\
\hline 12 & Faz queimadas & 0,027 & 0,332 & 0,034 & 0,038 & 0,742 & 0,665 \\
\hline \multirow[t]{5}{*}{13} & Prestação de serviços temporários & 0,020 & 0,333 & 0,110 & $-0,133$ & $-0,616$ & 0,520 \\
\hline & $\Sigma \mathrm{P}$ & 1,65 & 0,79 & 2,39 & 1,07 & 0,50 & \\
\hline & Raízes características & 2,22 & 2,06 & 1,87 & 1,31 & 1,27 & \\
\hline & Variância (\%) & 17,07 & 15,87 & 14,44 & 10,10 & 9,78 & \\
\hline & Variância cumulativa (\%) & 17,07 & 32,94 & 47,39 & 57,49 & 67,27 & \\
\hline
\end{tabular}

da atividade agrícola. Problemas semelhantes foram identificados por Luiz \& Silveira (2000) em estudo com os produtores rurais da microbacia de Taquara Branca (SP).

As variáveis "práticas agrícolas", "sinais de poluição das fontes hídricas e "faz queimadas" com cargas superiores a 0,$830 ; 0,560$ e 0,742 respectivamente, apontam para a necessidade de uma avaliação de como os recursos naturais estão sendo manejados. Em regiões secas, onde a disponibilidade de energia promove a mineralização da matéria orgânica, a queimada destrói a pouca matéria orgânica que permanece no solo (Duque, 1980). Foi observada, em poucos lotes, a existência de consórcios entre culturas preponderantes com leguminosas. A adoção de sistemas consorciado com leguminosas promovem o aumento da disponibilidade de nitrogênio para as culturas devido a fixação do mesmo por bactérias simbióticas. O aumento da produtividade de grãos em consórcio de milho e feijão em áreas irrigadas foi demonstrado por Cardoso et al. (1992).

Os fatores determinados pelo conjunto de variáveis que compõem cada componente estão ordenados de forma decrescente na Tabela 3, ou seja, o fator de ordem 1 é o que contém maior grau de importância na definição do índice de sustentabilidade. $\mathrm{O}$ fator 1 identifica a necessidade de mão-de-obra qualificada exigindo um maior grau de instrução e cursos especializados como uma decorrência natural da difusão de novos sistemas e técnicas de produção. Mangabeira et al. (2002) mostraram que o sistema de produção adotado pelos produtores rurais apresenta relação direta com o nível de desenvolvimento em que os mesmos se encontram. Por outro lado, é conhecido que o aspecto cultural, muito influente nestas populações, inibe ou dificulta, normalmente, o aperfeiçoamento através de cursos e treinamentos (Castelo Branco, 2003).

Durante a pesquisa, foi identificada em vários lotes irrigados, a não funcionalidade ou a ausência dos hidrômetros, deixando a deriva o consumo de água pela irrigação, sem uma preocupação maior na eficiência de uso da água. Uma outra conseqüência em cadeia é a insuficiência do sistema para abastecer os lotes agrícolas mais distantes, ocasionando, em determinados momentos, a falta de água para a irrigação das culturas. Souza et al. (2001) observaram uma atuação firme por parte do Distrito Irrigado Senador Nilo Coelho, através do corte de água, que proporcionou elevação nos índices de performance de pagamento dos usuários do sistema. 
ANDRADE, E.M. et al. Classificação da sustentabilidade das unidades de produção...

TABELA 3 - Denominação dos fatores associados às variáveis e os pesos (pi) associados aos indicadores de sustentabilidade. Perímetro Irrigado Araras Norte, Ceará. 2004.

\begin{tabular}{|c|c|c|c|}
\hline Ordem de fatores & Denominação do fator & Variáveis ou aspectos & Pesos $\left(p_{i}\right)$ \\
\hline \multirow{4}{*}{1} & \multirow{4}{*}{ Nível social e educacional da família } & Tamanho da família & 0,248 \\
\hline & & Número de filhos & 0,255 \\
\hline & & $\begin{array}{l}\text { Treinamento que gostaria de } \\
\text { receber }\end{array}$ & 0,092 \\
\hline & & Grau de Instrução & $-0,072$ \\
\hline \multirow{3}{*}{2} & \multirow{3}{*}{ Processo de comercialização } & $\begin{array}{l}\text { Tempo de exploração da } \\
\text { propriedade }\end{array}$ & 0,094 \\
\hline & & $\begin{array}{l}\text { Problemas por ocasião da } \\
\text { comercialização }\end{array}$ & $-0,133$ \\
\hline & & $\begin{array}{l}\text { Possui veículo para transporte } \\
\text { da produção }\end{array}$ & $-0,179$ \\
\hline \multirow{3}{*}{3} & \multirow{3}{*}{ Agricultura familiar } & $\begin{array}{l}\text { Continuidade das atividades } \\
\text { agrícolas }\end{array}$ & 0,139 \\
\hline & & $\begin{array}{l}\text { Os filhos participam das } \\
\text { atividades agrícolas }\end{array}$ & 0,134 \\
\hline & & $\begin{array}{l}\text { Prestação de serviços } \\
\text { temporários }\end{array}$ & $-0,001$ \\
\hline \multirow{3}{*}{4} & \multirow{3}{*}{ Adoção de técnicas de conservação } & Práticas agrícolas & 0,087 \\
\hline & & $\begin{array}{l}\text { Sinais de poluição das fontes } \\
\text { hídricas }\end{array}$ & 0,184 \\
\hline & & Faz queimadas & 0,152 \\
\hline Total & & & 1,000 \\
\hline
\end{tabular}

Os coeficientes ou pesos $\left(p_{\mathrm{f}}\right)$ atribuídos a cada variável que foram usados no cálculo do Índice de Sustentabilidade podem ser vistos na Tabela 3. Observa-se que os maiores pesos foram apresentados pelas variáveis "Número de filhos" e "Tamanho da família", as quais estão associadas ao fator "Nível social e educacional da família" (Tabela 3). Fossatti \& Freitas (2004) em estudos na região fumageira em Santa Cruz do Sul, RS encontraram resultados semelhantes, ou seja, o número de filhos e o grau de escolaridade foram identificados como os fatores determinantes da sustentabilidade dos agricultores. O terceiro e quarto maiores pesos foram atribuídos a variáveis que definem o fator "Adoção de técnicas de conservação" e "Processo de comercialização". Isso indica que, embora sem ter conhecimento profundo das técnicas, as famílias produtoras começam a se preocupar com a conservação dos recursos naturais, e com as limitações de transporte e comercialização da produção agrícola.

A Tabela 4 apresenta os índices de sustentabilidade gerados pela Equação 2 e a classificação das 47 unidades de produção agrícola no ranking do universo estudado. A hierarquização dos índices de sustentabilidade para cada unidade produtora foi feita a partir de índices absolutos. Os índices de sustentabilidade obtidos variaram no intervalo de 1,00 a 0,00 . Os menores valores significam níveis de maior sustentabilidade, enquanto os valores mais elevados significam níveis de maior insustentabilidade. A média global de sustentabilidade entre os produtores se situa em torno de 0,41 , apontando para uma condição de "sustentabilidade comprometida".

Para uma melhor visualização dos resultados, procedeu-se a uma partição das unidades em classes de acordo com sua posição relativa (Tabela 5). A partir da classificação adotada percebe-se que $27,7 \%$ das unidades de produção agrícola estudadas encontram-se numa situação de sustentabilidade equilibrada (Tabela 5 ) e que um percentual pouco menor das unidades de produção agrícola $(25,5 \%)$ ainda é considerada sustentável, mas tendo esta condição ameaçada, por qualquer um dos fatores contabilizados no índice. Outros $23,4 \%$ das unidades de produção agrícola registram uma sustentabilidade comprometida. Menos de um quarto das unidades de produção agrícolas estudadas são consideradas insustentáveis ou seriamente insustentáveis.

\section{CONCLUSÕES}

A sustentabilidade das unidades de produção agrícola estudadas foram determinadas principalmente por fatores que expressam o nível social e educacional da família, o processo de comercialização, a estabilidade da agricultura familiar e as técnicas de conservação do solo e da água. $\mathrm{O}$ índice de sustentabilidade desenvolvido classificou que $76,6 \%$ das unidades de produção agrícola apresentaram algum tipo de sustentabilidade, sendo que $27,7 \%$ do total mostraram-se sustentáveis. A relação causal estimada entre os fatores condicionantes e as variáveis mostrou que a única ocupação das famílias é a agricultura.

\section{AGRADECIMENTO}

Os autores agradecem ao Conselho Nacional de Desenvolvimento Científico e Tecnológico (CNPq) pelo apoio através da concessão de bolsas aos pesquisadores desse trabalho. 
ANDRADE, E.M. et al. Classificação da sustentabilidade das unidades de produção...

TABELA 4 - Índices de sustentabilidade e classificação das 47 unidades de produção agrícola estudadas. Perímetro Irrigado Araras Norte, Ceará, 2004.

\begin{tabular}{|c|c|c|c|c|c|}
\hline $\begin{array}{c}\text { Unidade } \\
\text { Produtiva }\end{array}$ & $I^{*}$ & Rank & $\begin{array}{c}\text { Unidade } \\
\text { Produtiva }\end{array}$ & IS* & Rank \\
\hline 26 & 0,000 & 1 & 39 & 0,380 & 24 \\
\hline 29 & 0,000 & 2 & 41 & 0,398 & 25 \\
\hline 10 & 0,000 & 3 & 23 & 0,402 & 26 \\
\hline 11 & 0,000 & 4 & 19 & 0,415 & 27 \\
\hline 32 & 0,051 & 5 & 20 & 0,444 & 28 \\
\hline 36 & 0,051 & 6 & 18 & 0,450 & 29 \\
\hline 38 & 0,076 & 7 & 14 & 0,533 & 30 \\
\hline 12 & 0,076 & 8 & 25 & 0,544 & 31 \\
\hline 37 & 0,088 & 9 & 22 & 0,570 & 32 \\
\hline 2 & 0,109 & 10 & 7 & 0,570 & 33 \\
\hline 28 & 0,180 & 11 & 5 & 0,579 & 34 \\
\hline 30 & 0,195 & 12 & 8 & 0,591 & 35 \\
\hline 31 & 0,196 & 13 & 13 & 0,600 & 36 \\
\hline 15 & 0,235 & 14 & 16 & 0,659 & 37 \\
\hline 42 & 0,241 & 15 & 17 & 0,702 & 38 \\
\hline 4 & 0,271 & 16 & 24 & 0,732 & 39 \\
\hline 1 & 0,278 & 17 & 21 & 0,734 & 40 \\
\hline 34 & 0,321 & 18 & 43 & 0,759 & 41 \\
\hline 40 & 0,341 & 19 & 46 & 0,765 & 42 \\
\hline 27 & 0,344 & 20 & 45 & 0,772 & 43 \\
\hline 44 & 0,364 & 21 & 35 & 0,802 & 44 \\
\hline 33 & 0,364 & 22 & 6 & 0,810 & 45 \\
\hline \multirow[t]{4}{*}{47} & 0,377 & 23 & 9 & 0,814 & 46 \\
\hline & & & 3 & 1,000 & 47 \\
\hline & & & Média & 0,413 & \\
\hline & & & Desvio Padrão & 0,270 & \\
\hline
\end{tabular}

TABELA 5 - Agrupamento das unidades de produção agrícola com relação à sustentabilidade. Perímetro Irrigado Araras Norte, Ceará, 2004.

\begin{tabular}{ccccc}
\hline Classes & Intervalos do IS & $\begin{array}{c}\text { Unidades de } \\
\text { produção agrícola }\end{array}$ & $\begin{array}{c}\text { Proporção } \\
(\%)\end{array}$ & $\begin{array}{c}\text { Proporção } \\
\text { Acumulada }\end{array}$ \\
\hline Sustentável & IS $<0,20$ & 13 & 27,7 & 27,7 \\
Sustentabilidade Ameaçada & $0,20 \leq$ IS $<0,40$ & 12 & 25,5 & 53,2 \\
$\begin{array}{c}\text { Sustentabilidade } \\
\text { Comprometida } \\
\text { Insustentável }\end{array}$ & $0,40 \leq$ IS $<0,60$ & 11 & 23,4 & 76,6 \\
Seriamente Insustentável & $0,60 \leq$ IS $<0,80$ & 7 & 14,9 & 91,5 \\
\hline TOTAL & IS $\geq 0,80$ & 4 & 8,5 & 100,0 \\
\hline
\end{tabular}

${ }^{1}$ IS - Índice de sustentabilidade

\section{REFERÊNCIAS}

1. ANDRADE, E. M. et al. Impacto da lixiviação de nitrato e cloreto no lençol freático sob condições de cultivo irrigado. Revista Ciência Rural, v. 9, n. 1, 2009. Aceito para publicação.

2. ANDREWS, S. S.; KARLEN, D. L.; MITCHELL, J. P. A comparison of soil quality indexing methods for vegetable production systems in Northern California. Agriculture, Ecosystems and Environment, v. 90, n. 1, p. 25-45, 2002.

3. BARRETO, R. C. S.; KHAN, A. S.; LIMA. P. V. P. S. Sustentabilidade dos assentamentos no município de Caucaia - CE. Revista de Economia e Sociologia Rural, v. 43, n. 2, p. 225-247. 2005.

4. CASTELO BRANCO, M. Avaliação do conhecimento do rótulo dos inseticidas por agricultores em uma área agrícola do Distrito Federal. Revista Horticultura Brasileira, v. 21, n. 3, p. 570-573, 2003.

5. CARDOSO, M. J. et al. População de plantas no consórcio milho $x$ feijão macassar sob regimes de sequeiro e irrigada. Revista Ciência Agronômica, v. 23, n. 1, p. 21-31, 1992.

6. DEPARTAMENTO NACIONAL DE OBRAS CONTRA SECA (DNOCS). Perímetros irrigados. Disponível em: <http:// www.dnocs.gov.br/ dnocs/doc/canais/perimetros_irrigados/ce/araras_norte.html>. 2005. Acesso em: 28 dez. 2007. 
ANDRADE, E.M. et al. Classificação da sustentabilidade das unidades de produção...

7. DEPARTAMENTO NACIONAL DE OBRAS CONTRA SECA (DNOCS). Plano diretor do Vale do Acaraú: Pedologia. Fortaleza, 1977. v. 2, 96 p.

8. DILLON, W. R.; GOLDSTEIN, M. Multivariate analysis: methodos and applications. New York: John Wiley \& Sons, 1984. $587 \mathrm{p}$.

9. DUQUE, J. G. Solo e água no polígono das secas. 5. ed. Mossoró: Escola Superior de Agricultura de Mossoró, 1980. $273 \mathrm{p}$.

10. FOSSATTI, D. M.; FREITAS, C. A. O caráter familiar da atividade fumageira em Santa Cruz do Sul/ RS. In: CONGRESSO BRASILEIRO DE ECONOMIA E SOCIOLOGIA RURAL, 17., Cuiabá, 2004. Anais... Cuiabá: Passo, 2004.1 CD-ROM.

11. GALLOPÍN, G. C. Indicators and their use: information for decision-making. In: MOLDAN, B; BILLHARZ, S. (Ed.). Sustainability indicators: report of the project on indicators of sustainable development. New York: John Wiley \& Sons, 1997. Cap. 1, p. 13-27.

12. GIRÃO, E. G. et al. Seleção dos indicadores de qualidade de água no Rio Jaibaras pelo emprego da análise da componente principal. Revista Ciência Agronômica, v. 38, n. 1, p. 17-24, 2007.

13. HAIR JUNIOR, J. F. et al. Análise multivariada de dados. Tradução de Adonai Schlup Sant'Anna e Anselmo Chaves Neto. 5. ed. Porto Alegre: Bookman, 2005. 593 p.

14. LUIZ, A. J. B.; SILVEIRA, M. A. Diagnóstico rápido e dialogado em estudos de desenvolvimento rural sustentável. Revista Pesquisa Agropecuária Brasileira, v. 35, n. 1, p. 83-91, 2000.

15. MANGABEIRA, J. A. et al. Tipificação de sistemas de produção rural: a abordagem da análise de correspondência múltipla em Machadinho d'Oeste-RO. Campinas: Embrapa Monitoramento por Satélite, 2002. 30 p. (Circular Técnica, 8).

16. MARZALL, K.; ALMEIDA, J. Parâmetros e indicadores de sustentabilidade na agricultura: limites, potencialidades e significado no contexto do desenvolvimento rural. Extensão Rural, n. 5, p. 25-38, 1998.

17. MASERA, O.; ASTIER, M.; LOPES, R. S. Sustentabilidad y manejo de recursos naturales: el marco de evaluación (MESMIS). Mexico: Mundi-Prensa, 1999. 107 p.

18. MELO, A. S. S. A. Estimação de um índice de agricultura sustentável: o caso da área irrigada do Vale do Submédio São Francisco. 1999. 167 f. Tese (Doutorado em Economia) - Curso de Pós-Graduação em Economia, Universidade Federal de Pernambuco, Recife, 1999.

19. PALÁCIO, H. A. Q. Índice de qualidade das águas na parte baixa da bacia hidrográfica do rio Trussu Ceará. 2004. 96 f. Dissertação (Mestrado em Irrigação e Drenagem) - Curso de Pós-Graduação em Irrigação e Drenagem, Universidade Federal do Ceará, Fortaleza, 2004.

20. SANDS, G. R.; PODMORE, T. H. A generalized environmental sustainability index for agricultural systems. Agriculture, Ecosystems and Environment, v. 79, n. 1, p. 29-41, 2000.

21. SILVEIRA, S. S.; ANDRADE, E. M. Análise de componente principal na investigação da estrutura multivariada da evapotranspiração. Engenharia Agrícola, v. 22, n. 2, p. 171-177, 2002.

22. SOUZA, G. H. F. et al. Desempenho do Distrito de Irrigação Senador Nilo Coelho. Revista Brasileira de Engenharia Agrícola e Ambiental, v. 5, n. 2, p. 204-209, 2001.

Recebido em 19/03/2008 Aceito em 03/12/2008 